\title{
Ionic Liquids Confined in Silica Ionogels: Structural, Thermal, and Dynamical Behaviors
}

\author{
Subhankur Mitra ${ }^{1,2}$, Carole Cerclier ${ }^{3}$, Quentin Berrod ${ }^{1}$, Filippo Ferdeghini ${ }^{1,4}$, \\ Rodrigo de Oliveira-Silva ${ }^{1}$, Patrick Judeinstein ${ }^{1}$, Jean le Bideau ${ }^{3}$ and Jean-Marc Zanotti ${ }^{1, *}$ \\ 1 Laboratoire Léon Brillouin, CEA-CNRS, CEA Saclay, 91191 Gif-sur-Yvette Cedex, France; \\ mitra_subhankur@yahoo.co.in (S.M.); Quentin.Berrod@cea.fr (Q.B.); filippo.ferdeghini@ece.fr (F.F.); \\ rodrigo.de-oliveira-silva@cea.fr (R.d.O.-S.); Patrick.Judeinstein@cea.fr (P.J.) \\ 2 Solid State Physics Division, Bhabha Atomic Research Centre, Mumbai 400 085, India \\ 3 Institut des Matériaux Jean Rouxel (IMN), Université de Nantes-CNRS, 2 Rue de la Houssinère BP 32229, \\ 44322 Nantes Cedex 3, France; carole.cerclier@gmail.com (C.C.); Jean.LeBideau@cnrs-imn.fr (J.1.B.) \\ 4 Laboratoire des Interfaces Complexes et de l'Organisation Nanométrique, ECE-Paris Ecole d'Ingénieurs, \\ 37 Quai de Grenelle, 75015 Paris, France \\ * Correspondence: jmzanotti@cea.fr; Tel.: +33-01-6908-9701
}

Academic Editors: Giancarlo Franzese, Ivan Latella and Miguel Rubi

Received: 31 January 2017; Accepted: 20 March 2017; Published: 24 March 2017

\begin{abstract}
Ionogels are porous monoliths providing nanometer-scale confinement of an ionic liquid within an oxide network. Various dynamic parameters and the detailed nature of phase transitions were investigated by using a neutron scattering technique, giving smaller time and space scales compared to earlier results from other techniques. By investigating the nature of the hydrogen mean square displacement (local mobility), qualitative information on diffusion and different phase transitions were obtained. The results presented herein show similar short-time molecular dynamics between pristine ionic liquids and confined ionic liquids through residence time and diffusion coefficient values, thus, explaining in depth the good ionic conductivity of ionogels.
\end{abstract}

Keywords: ionic liquid; confinement; quasi-elastic neutron scattering; phase transition; mobility

\section{Introduction}

Room temperature ionic liquids (ILs) are salts of weakly-interacting organic cations and anions which melt below approximately $100{ }^{\circ} \mathrm{C}$, and with peculiar properties, such as negligible vapor pressure, thermal stability, non-flammability, high ionic conductivity, or even compatibility with biological systems. Thus, numerous studies have been focused on ILs; for instance, their use in biocatalysis [1] or gas storage [2]. Currently, their uses are driving materials science for energy, for instance, as lithium batteries [3,4], supercapacitors [5], and solar and fuel cells [6]. More recent studies are emerging in the biomaterials field. In addition to the goal of taking advantage of the properties of ILs in materials, their liquid nature limits their use when all-solid devices are desired. Thus, taking advantage of IL properties in solid-state materials remains a major challenge [7]. To this aim, several approaches are under investigation through grafting on supports [8], swelling in polymers [9], confinement in carbon nanotube membranes [10], and impregnation on preliminarily-prepared oxide particles. For the last few years, we have developed an original sol-gel route carried out in ILs that leads to the nano-confinement of ILs in an open 3-D interconnected mesoporous oxide network: so-called ionogels. In classical sol-gel processing, the targeted porous metal oxide solid is obtained after drying and, thus, removal of the solvent. The use of IL permits us to regard the gel itself, confining the IL, as a stable solid-liquid system. It has been demonstrated that these solid ionogels could present IL 
properties, such as high ionic conductivity $[11,12]$ and liquid-like efficient chromophore solvation within a monolith, thus opening new fields for IL properties in all-solid devices.

Extensive investigations were also carried out to ascertain the stability towards solvents, to characterize the phase transitions and the dynamics of these ILs entrapped in ionogels [11,12]. Dynamic scanning calorimetry (DSC) experiments revealed the dramatic effect of confinement on the phase transitions of the IL within the ionogels. It has been observed that both the phase transitions related to cold crystallization and melting disappears when pore size induced by the IL content is decreased. Although the melting is observed in cases of relatively less-confined IL (i.e., larger pore size), the melting temperature $(\mathrm{Tm})$ is found to be shifted to the lower temperature than for the pristine liquid, whereas the cold-crystallization temperature (Tcc) was observed at a higher value. This is explained in terms of simple mean field theory, which relates the Tm decrease to the decrease of the number of nearest neighbors in the case of the confined IL. Solid state ${ }^{1} \mathrm{H}$ NMR spectroscopy indicated that the confined IL still keeps liquid-like molecular motions [13]. It was seen that the line width does not change much if one goes from a spinning rate of $400 \mathrm{~Hz}$ to $3 \mathrm{KHz}$. However, line width was seen to be increased in the case of ILs confined within smaller pores, which suggested that there was a slowdown in the dynamics occurring when confinement increases (pore size decreases). The ionogels showed a conductivity of the same order of magnitude as the pristine ionic liquid. Arrhenius plots $[11,12]$ $\log (\sigma T)$ vs. $\left(1 \times 10^{3}\right) / \mathrm{T}$ showed a break-slope around $330 \mathrm{~K}$, reflecting lower activation energies at temperatures higher than $330 \mathrm{~K}$. Such a break in the activation energy value around $330 \mathrm{~K}$ suggests that a thermodynamical transition occurs at $330 \mathrm{~K}$, modifying the molecular arrangement from ion pairs to single ions. To investigate more in depth these phase transitions and compare the findings with the results obtained using other methods, we have, herein, performed a neutron scattering study.

\section{Materials and Methods}

\subsection{Ionogels}

1-butyl-3-methylimidazolium bis(trifluromethylsulfonyl) imide [BMI][TFSI] was synthesized as a colorless transparent liquid according to the literature [14]. 1-methylimidazole was reacted with a slight excess of 1-bromobutane in a round-bottom flask and left refluxing for three hours to produce 1-butyl-3-methylimidazolium bromide [BMI][Br]. The corresponding [TFSI] salt was then prepared by metathesis of $[\mathrm{BMI}][\mathrm{Br}]$ in water with sodium bis(trifluromethylsulfonyl) amide. Ionogels were prepared as previously published [15]: tetramethoxysilane (TMOS) and/or methyltrimethoxysilane (MTMS) (purchased from Alfa Aesar, Tewksbury, MA, USA), were added to a solution of formic acid (FA) in [BMI][TFSI] (molar ratio IL/TMOS/FA or IL/(TMOS + MTMS)/FA = 0.5 (and 0.25) $/ 1 / 7.8$ (referred as $x=0.5$ (and 0.25))). Other ionogels were made with a molar ratio IL/TMOS/FA or $\mathrm{IL} /(\mathrm{TMOS}+\mathrm{MTMS}) / \mathrm{FA}=1 / 1 / 7.8$ (referred as $x=1$ ), resulting in larger pores; their characterization is shown elsewhere [11,12]. After a short stirring, gelation occurred within $1 \mathrm{~h}$ for a TMOS ionogel, or within $36 \mathrm{~h}$ for a TMOS/MTMS ionogel. Aging takes place overnight in a bath at a room temperature with exposure to ultrasound (12 W power, $2 \mathrm{~s}$ pulse). Pristine IL, [BMI][TFSI], and deuterated IL, [BMID][TFSI], were compared by neutron scattering experiment.

\subsection{Calorimetry}

Measurements of phase-transition temperatures were done with a Netzsch differential scanning calorimeter (model 204 F1 Phoenix, Burlington, MA, USA), and the data were evaluated using Netzsch Proteus Thermal Analysis software version 4.8.1. Samples of 10-15 mg were placed in a hermetically sealed aluminum pan; an empty pan was used as a reference. Pans were exposed to a $\mathrm{N}_{2}$ flow atmosphere. Measurements for the melting, crystallization, and glass-transition temperatures were determined by heating the sample at $+100^{\circ} \mathrm{C}$, then cooling the sample to $-120^{\circ} \mathrm{C}$ at a rate of $50{ }^{\circ} \mathrm{C} / \mathrm{min}$, followed by heating from -120 to $150{ }^{\circ} \mathrm{C}$ at a rate of $10{ }^{\circ} \mathrm{C} / \mathrm{min}$. The glass transition was determined 
at the midpoint of a heat-capacity change, whereas the melting and crystallization temperatures were determined at the onset of the transition.

\subsection{Quasi-Elastic Neutron Scattering (QENS)}

The QENS study is performed using the inelastic time of flight neutron spectrometer, MIBEMOL (detailed information on Mibemol can be found at [16]) at the Laboratoire Léon Brillouin, Saclay, France. The monochromatization of the incident beam is achieved by a system of six choppers. Scattered neutrons are detected by arrays of $\mathrm{He}^{3}$ neutron detectors (32 detectors within the angular range $12^{\circ}<2 \theta<32^{\circ}$ and about 400 detectors within the angular range $32^{\circ}<2 \theta<147^{\circ}$ ). Two instrumental configurations have been used with $6 \AA$ and $5.2 \AA$ incident wavelengths showing, respectively, full width at half maximum (FWHM) elastic energy resolutions of $R_{F W H M}=96 \mu \mathrm{eV}$ and $150 \mu \mathrm{eV}$. An elastic scan is performed from 150 to $440 \mathrm{~K}$. This elastic scan is aimed to observe the behavior of mean square displacements with temperature. A long-time run is also performed in the temperature range 150 to $440 \mathrm{~K}$ in case of the methylated ionogel sample. An elastic scan is also performed using the QENS spectrometer, at IPNS, Argonne National Laboratory, Argonne, IL, USA. QENS [17] is a so-called inverted geometry spectrometer where the sample is enlighten by a polychromatic neutron beam. Pyrolytic graphite analyzers are placed in between the sample and the $146 \mathrm{He}^{3}$ detectors so that only one wavelength is detected. The energy resolution of QENS is constant $R_{F W H M}=80 \mu \mathrm{eV}$. While the technical principles of MIBEMOL and QENS are different (respectively, direct vs. indirect geometry), both instrument directly access the double differential cross section $\frac{\partial^{2} \sigma}{\partial \Omega \partial E}$ which gives the probability that a neutron with incident energy $E_{0}$, leaves the sample in the solid angle $\mathrm{d} \Omega$ about the direction $\Omega$ with an energy change comprised between $\hbar \omega=E-E_{0}$ and $\hbar(\omega+d \omega)$. This double differential cross section can be written as [18]:

$$
\frac{\partial^{2} \sigma}{\partial \Omega \partial E}=A \frac{k}{k_{0}}\left[\sigma_{i n c} S_{i n c}(Q, \omega)+\sigma_{c o h} S_{c o h}(Q, \omega)\right]
$$

where $A$ stands for a constant number depending on the number of nuclei in the sample, and $k$ and $k_{0}$ are the final and incident wave vectors of the scattered and incident neutrons, respectively. $Q$ is the neutron wave vector transfer $\left(k-k_{0}\right)$. Parameters $\sigma_{c o h}$ and $\sigma_{\text {inc }}$ are the coherent and incoherent cross-sections of the sample under study. $S_{c o h}(Q, \omega)$ and $S_{\text {inc }}(Q, \omega)$ are defined as the coherent and incoherent scattering functions, respectively. The incoherent scattering function, $S_{i n c}(Q, \omega)$ is the Fourier transform of self-intermediate scattering function $I_{S}(Q, t)$, which is written as [18]:

$$
I_{S}(Q, t)=\sum_{i}\left\langle\exp \left(i Q r_{i}(t)\right) \cdot \exp \left(-i Q r_{i}(0)\right)\right\rangle
$$

where $r_{i}(t)$ and $r_{i}(0)$ are the position of the $i$ th particle at time $t$ and 0 , respectively. The coherent scattering function, $S_{c o h}(Q, \omega)$ is the Fourier transform of the intermediate scattering function $I(Q, t)$, which can be written as:

$$
I(Q, t)=\sum_{i, j}\left\langle\exp \left(i Q r_{i}(t)\right) \cdot \exp \left(-i Q r_{j}(0)\right)\right\rangle
$$

On one hand, as can be seen from Equation (3), the coherent scattering function contains information on interference effects arising from spatial correlations of the nuclei in the system: therefore, it contains collective information about the sample. On the other hand, the incoherent scattering function, Equation (2), contains information only on the dynamics of single atoms (self-diffusion) which may be investigated via energy analysis of the scattered neutrons. In case of hydrogenous sample, $\sigma_{i n c} \gg \sigma_{c o h}$ and Equation (1) can be written as:

$$
\frac{\partial^{2} \sigma}{\partial \Omega \partial E}=A \frac{k}{k_{0}}\left[\sigma_{i n c} S_{i n c}(Q, \omega)\right]
$$


It is seen that these scattering functions depend purely on the structural and dynamical properties of the scattering sample. Therefore, by analyzing the scattered neutron as a function of $Q$ and $\omega$, one can have the detail information about the structure or the dynamics of the scattered sample.

Considering that the particle can undergo different dynamical behaviors (e.g., vibrational, rotational or translational motion) simultaneously and these different dynamics are independent of each other, incoherent scattering function can be written as [18]:

$$
S_{\text {inc }}(Q, \omega)=\exp \left(-\frac{Q^{2}<u^{2}>}{3}\right) S_{\text {rot }}(Q, \omega) \otimes S_{\text {trans }}(Q, \omega)
$$

Here, $<u^{2}>$ denotes the mean square displacement and the exponential term arises mainly from the vibrational motion. The final scattering function (scattered neutron intensity) is, thus, the convolution product between scattering functions arising purely from the rotational motion and the translational motion. However, in the case of the quasi-elastic neutron scattering experiment, in the absence of any particular insight of the possible dynamical motions present in the system, one can write the incoherent scattering function in a more general form such as:

$$
S_{i n c}(Q, \omega)=\exp \left(-\frac{Q^{2}<u^{2}>}{3}\right)[A(Q) \delta(\omega)+(1-A(Q)) L(Q, \omega)]
$$

The term $A(Q) \delta(\omega)$ is generally called the elastic contribution and the $(1-A(Q)) L(Q, \omega)$ is known as the quasi-elastic component. $L(Q, \omega)$ denotes a Lorentzian with half width at half maxima (HWHM), $\Gamma(Q)$. The fraction of elastic component in the total spectra is known as the elastic incoherent structure factor (EISF) and gives information about spatial distribution of the particle in $Q$ space and can be used to extract information due to localized motions (e.g., rotational or motion under confinement) present in the system. Therefore, $A(Q)$ in Equation (6) can be identified as the EISF. $\Gamma(Q)$ is inversely proportional to the characteristic time of the dynamics and can also be used to find the diffusion constant for the diffusive motions present in the system.

To increase the signal to noise ratio, neutron counts detected in adjacent detectors are summed and the corresponding detection angle $2 \theta$ (or equivalently $Q$ ) is the average of the angle of the detectors considered. This is the so-called "detector grouping" procedure. The analysis is, therefore, made on a reduced number, typically ten, detector groups.

Data treatment (empty cell subtraction, vanadium normalization, detectors grouping) and data fitting have been performed using Qensh software (Qensh software can be found at www-llb.cea.fr/ en/Phocea/Page/index.php?id=21 with detailed information).

\subsection{Diffraction}

The diffraction measurements are performed using the two-axis powder diffractometer G6-1 (detailed information on G6-1 can be found at [19]) at LLB, France. The incident wavelength of $4.749 \AA$ is selected by a graphite monochromator. The detector can rotate around the sample axis from 0 to $110^{\circ}$ ( $Q$-range [0.15-1.5] $\AA^{-1}$ ) with a variable resolution from $2 \theta \sim 2^{\circ}$ (detector in the direct beam) to $2 \theta$ $\sim 150^{\circ}$ (detector at $110^{\circ}$ ).

\section{Results and Discussion}

The aim of this paper is to examine the nature of phase transitions in ionogels using a neutron scattering experiment and compare the findings with the results obtained using other methods. One such method used earlier to find the nature of phase transitions is differential scanning calorimetry (DSC). DSC experiments were performed on ionogels with different contents of IL [11,12]. In Figure 1, the results of DSC experiments are shown for pristine liquid (inset) and for different ionogels. The DSC of the pristine IL exhibited, upon heating after a fast cooling, a glass-transition temperature (Tg) at $186 \mathrm{~K}$ (the compound passed from the glass state to a "subcooled liquid" phase), an exothermic 
cold crystallization $(T \mathrm{cc})$ at $228 \mathrm{~K}$, and a subsequent endothermic melting $(T \mathrm{~m})$ at $270 \mathrm{~K}$. In ionogels, although the glass transition was observed in all cases (whatever the molar ratio $x=\mathrm{IL} / \mathrm{Si}$ was), some occurrence of crystallization and melting were observed for $x=1$, but not for $x=0.25$ and 0.50 (in either pure silica ionogel or methyl-modified ionogel). Moreover, for ionogels with $x=1$, the temperature of the first-order phase transition (melting) was clearly shifted to a lower value. Table 1 gives the comprehensive values of transition temperatures and enthalpies obtained through DSC measurements for pure IL, non-methylated ionogel and methylated ionogels, and a subsequent molar fraction of confined IL, which undergoes crystallization and melting. These values corroborate those obtained in the case of similar ILs [BMI] $\left[\mathrm{CF}_{3} \mathrm{SO}_{3}\right]$ or [BMI][TFSI] confined in controlled pore glasses (CPGs) [20].

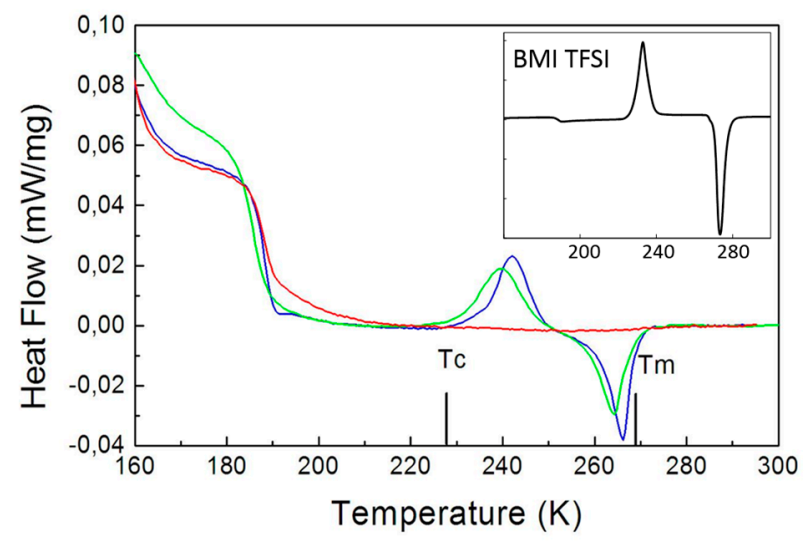

Figure 1. DSC traces of two silica based ionogels ( $x=0.5$, red; $x=1$, blue) and one methylated ionogel (TMOS/MTMOS $1 / 1 ; x=1$, green). Tc and Tm are crystallization and melting temperatures, respectively. Vertical bars on the abscissa represent $T \mathrm{c}$ and $T \mathrm{~m}$ for the pristine IL [BMI][TFSI] (inset).

Table 1. Values of transition temperatures and enthalpies obtained from DSC studies for pure IL, non-methylated (TMOS) and methylated (TMOS/MTMS) $x=1$ ionogels (crystallization and melting are not observed in $x=0.25$ and $x=0.5$ ionogels).

\begin{tabular}{ccccccc}
\hline & $\boldsymbol{x}$ & $\boldsymbol{T}_{\mathbf{c}}(\mathbf{K})$ & $\boldsymbol{T}_{\mathbf{f}}(\mathbf{K})$ & $\begin{array}{c}\boldsymbol{H}_{\mathbf{c}} \\
\left(\mathbf{J} \cdot \mathbf{m o l}^{-\mathbf{1}} \mathbf{)}\right.\end{array}$ & $\begin{array}{c}\boldsymbol{H}_{\mathbf{f}} \\
\left(\mathbf{J} \cdot \mathbf{m o l}^{-\mathbf{1}} \mathbf{)}\right.\end{array}$ & $\begin{array}{c}\text { Crystallization } \\
\text { (Molar Ratio) }\end{array}$ \\
\hline BMIM TFSI & & 228 & 270 & 17,800 & 21,800 & \\
TMOS & 1 & 242 & 266 & 656 & 532 & 3.7 \\
TMOS/MTMS & 1 & 240 & 264 & 570 & 538 & 3.2 \\
\hline
\end{tabular}

In case of an elastic scan using neutrons, quasielastic spectra are recorded with a fixed instrumental resolution as a function of the temperature. The idea is to monitor the elastic intensity (around zero energy transfer) as a function of temperature. The statistics of counting for a particular spectrum at a particular temperature does not need to be very good since the relevant information comes only from the elastic intensity and not from the whole spectrum. Therefore, short-time quasi-elastic measurements are recorded at various temperatures. At higher temperature, the dynamics of the scatterer in the sample are enhanced and the intensity of the elastic contribution decreases since more neutrons are scattered with a higher energy transfer. Therefore, monitoring the elastic intensity with a function of temperature gives qualitative information about a possible dynamic change in the system with temperature. In this case, elastic intensity (at $\omega=0)$ can be assumed as:

$$
I_{e l}(Q)=A \cdot \exp \left(-Q^{2} \cdot<u^{2}>\right)
$$


Here, mean square displacement $\left\langle u^{2}\right\rangle$ is a function of temperature. For a set of quantized harmonic oscillators, the mean square displacement is given by [21]:

$$
<u^{2}>=\frac{k_{B} \theta}{2 K \tan h\left(\frac{\theta}{2 T}\right)}
$$

where $\theta=\omega_{0} . \hbar / k_{B}$ is the Debye temperature, $\omega_{0}$ and $K$ stand for the frequency and force constant of the oscillator, respectively. For, $T<\theta / 2$, the mean square displacement is almost a constant equal to zero-point fluctuations, i.e., $k_{B} \theta / K$. For $T>\theta / 2$, the mean square displacements linearly increases with the temperature, and approximately takes the form $k_{B} T / K$. However, when the scatterer experiences an anharmonic potential at higher temperature (may be due to phase transition or any other modification of dynamic motions coming into play, like the ion pairs to single ion behavior for instance), the mean square displacement deviates from linearity with temperature. It may also happen that it still continues to be linear, but that changes occur in the slope's values due to phase transition or because of the onset of other dynamic contributions. This is mainly because the effective force constant changes when other dynamic contributions comes into play. Therefore, a modification of linearity in the mean square displacement curve with temperature suggests a possible phase transition or onset of extra dynamic contributions.

Figure 2 shows the behavior of the elastic intensity extracted from quasi-elastic neutron scattering (QENS) spectra versus $Q^{2}$ for the non-methylated $x=0.5$ ionogel at $393 \mathrm{~K}$ using the Mibemol spectrometer. Variation of the elastic intensity suggests a Gaussian behavior with $Q$. Elastic intensity data are fitted with Equation (7) to extract the mean square displacement. It may be noted that the obtained mean square displacements are more qualitative in nature than quantitative, which fits the requirements of our purpose: to compare the transitions with temperature observed by neutron scattering at the molecular scale, to the transitions observed by other techniques. A mean square displacement of $0.26 \AA^{2}$ is extracted from the slope of the elastic intensity vs. $Q^{2}$ as shown in Figure 2. The same procedure is applied for each temperature probed (during the short quasielastic scans) to extract the temperature dependence of the mean square displacement, as shown in Figure 3.

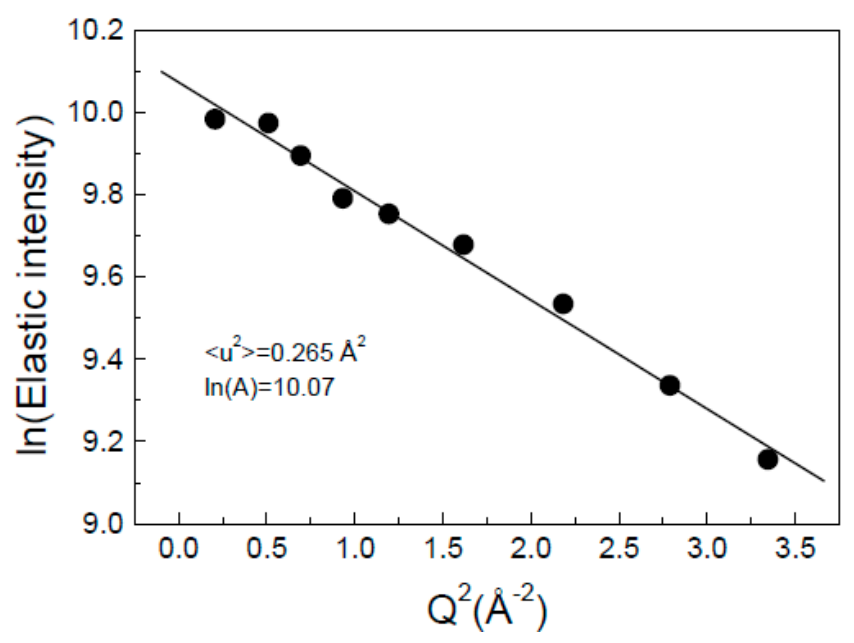

Figure 2. Variation of elastic intensity with $Q^{2}$ shown here for ionogel non-methylated $x=0.5$ at $393 \mathrm{~K}$. Mean square displacement is obtained by fitting Equation (7) with the data.

It is to be noted that absolute values of $\left\langle u^{2}>\right.$ using two different instruments could be different as the two experiments were performed using completely different sets of parameters. Nonetheless, as can be seen, both the results match rather well qualitatively. As explained with Equation (8) at very low temperature (well below the Debye temperature) the mean square displacements are almost constant and follow zero-point fluctuations. It can be observed in the IPNS data, that at low temperature, 
$<u^{2}>$ is almost constant (very small) and then it increases linearly with temperature. It can be seen that around $200 \mathrm{~K}$, there is a modification of the slope of $<u^{2}>$ vs. temperature curve suggesting a glass transition (which was already observed in DSC measurements as shown in Figure 1). From the Mibemol data, also, it can be clearly seen that there is a definite phase transition around $225 \mathrm{~K}$ which can be identified as the glass transition, also observed around $228 \mathrm{~K}$ by DSC (discussed above). In addition to this, the Mibemol data shows that there is a modification of the slope at around $275 \mathrm{~K}$; similarly, a modification is also noted in IPNS data around $250 \mathrm{~K}$. This transition is to be identified as the endothermic melting $(\mathrm{Tm})$ seen in DSC experiment at $269 \mathrm{~K}$. From the Mibemol data, another transition is observed at around $360 \mathrm{~K}$. Although DSC study was performed up to that temperature, nothing was visible; nevertheless, complex impedance spectroscopy studies indicated a change of slope in the Arrhenius plot $\left(\log (\sigma T)\right.$ vs. $\left.\left(1 \times 10^{3}\right) / T\right)$ around $330 \mathrm{~K}[11,12]$.

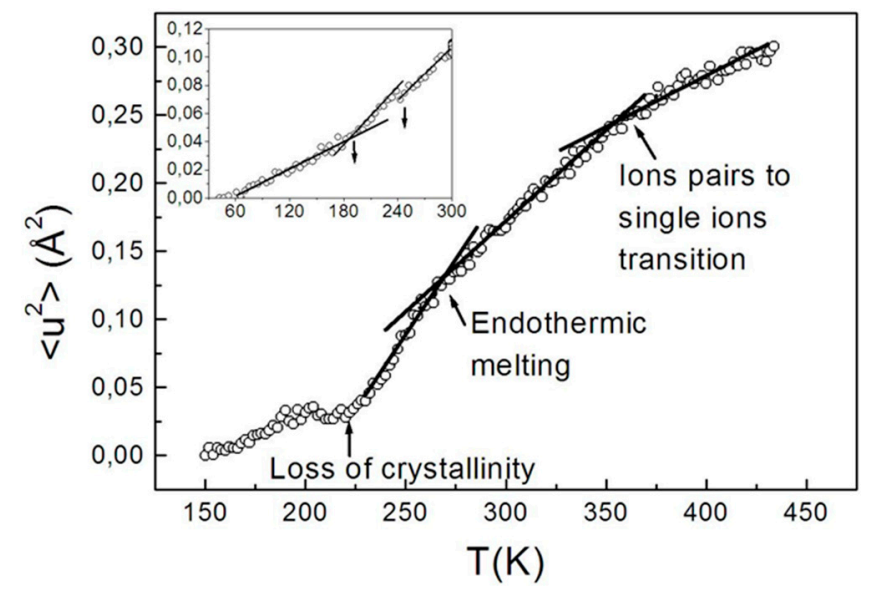

Figure 3. Variation of mean square displacement with temperature, in case of a methylated $x=0.5$ ionogel. Mibemol spectrometer (at LLB) is used for the experiment. Inset: data obtained using QENS spectrometer at IPNS.

Nanometer-scale structuring in room-temperature ILs has been observed using molecular dynamics simulation [22]. Later Triolo et al. have experimentally shown that there exist nanoscale heterogeneities in similar types of ILs [23]. These heterogeneities are of the order of a few nanometers and their size is proportional to the alkyl chain length. Our preliminary experiment also showed that either similar domain structure exists in [BMI][TFSI], or ion pairs to single ion transition occurs. When increasing the temperature, these nanoscale domains or ion pairing disappear. There is a temperature at which the Coulomb vs. Van der Waals interactions clearly put a disadvantage in the overall attractive energy which tight any correlated domains or pairs [24-26]. Thus, breaking of these correlations possibly gives rise to the decreased activation energies which can be clearly seen around $340 \mathrm{~K}$ in the Arrhenius plot of the ionic conductivity [11,12]. The slope change in $<u^{2}>$ around $360 \mathrm{~K}$, as seen in Figure 3, can be correlated with this transition as well.

One can also exploit the diffraction pattern of the sample from an inelastic neutron scattering experiment, by plotting elastic intensities as a function of $Q$. Variation of elastic intensities with $Q$ are estimated from the long quasi-elastic runs (using Mibemol) for various temperatures and are plotted in Figure S1. As can be seen from Figure S1, only one prominent diffraction peak appears around $1.45 \AA^{-1}$. However, since the spatial resolution for measuring a diffraction pattern is poor, and the number of detectors are fewer in an inelastic instrument, other peaks are not particularly prominent in this spectrum. Therefore, diffraction data is taken using diffractometer G61 at LLB, which has a very good resolution in order to identify various Bragg peaks. Diffraction data from the ionogel as a function of temperature are shown in Figure 4 . It is seen that the data at $150 \mathrm{~K}$ does not exhibit any diffraction peaks. This is in accordance with our earlier results from DSC and mean square displacements studies 
which showed that the glass transition temperature $(\mathrm{Tg})$ is around $186 \mathrm{~K}$. Let us remind here that the sample was heated at $100{ }^{\circ} \mathrm{C}$ prior to its fast cooling. Thus, according to the DSC results (at least seen for $x=1$ ionogel), from $200 \mathrm{~K}$ upwards, some diffraction peaks appear before vanishing after an endothermic melting $(T \mathrm{~m}=269 \mathrm{~K})$ as seen with the data at $T=280 \mathrm{~K}$ and RT which showed no diffraction peaks. There is also a signature of a surface scattering observed at small angle (or $Q$ ) and can be described by the Porod's law which predicts that the scattering at low $Q$ values is determined from the formula, $I(Q) \propto Q^{-4}$. Few peak positions and the corresponding peak intensities are given in the Table S1. Figure 5 shows the intensity evolution of various peaks with temperature. It can be seen that all the peaks are systematically vanishing when the temperature increase. It is noteworthy to that if crystallization and subsequent melting was seen by DSC for $x=1$ ionogel, it was not seen for $x=0.5$ ionogel (Table 1), which means that less than around 3\% mol of confined IL experienced crystallization in this last ionogel; thus, neutron diffraction allowed us to see more precisely that even in the $x=0.5$ ionogel, a small part (maybe less than $1 \%$ ) of confined IL experienced crystallization and melting.

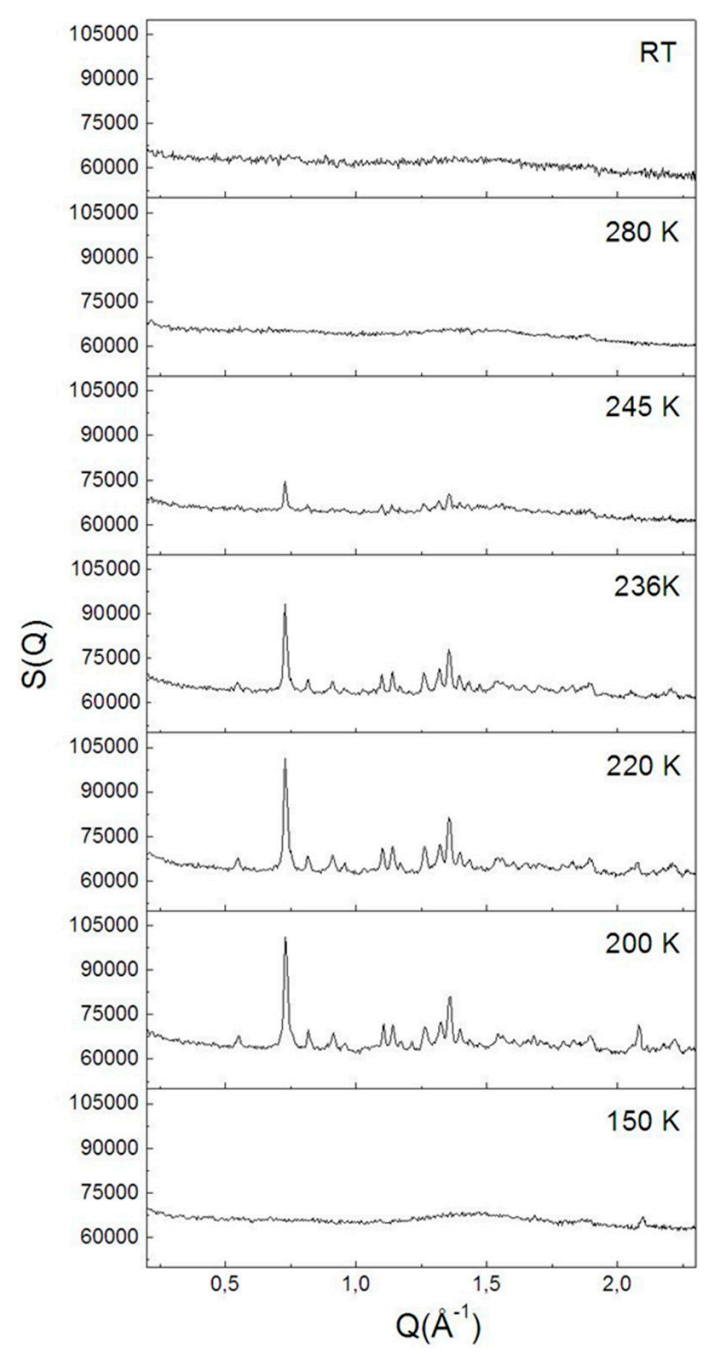

Figure 4. Diffraction data at different temperatures for a methylated $x=0.5$ ionogel.

Quasi-elastic spectra $(S(Q, \omega))$ recorded for various samples at high temperature $(T=393 \mathrm{~K})$ are shown in Figure 6. It is seen that the overall width of the quasi-elastic signal, which is related to the time scale of the dynamics, is smallest for methyl-modified ionogel. The pristine protonated IL's signal is broader than that of ionogels and the deuterated IL showed the largest signal width. This last point can be simply understood by the isotopic effect of the smaller molecular weight of 
the parent protonated imidazolium. Additionally, the effect of confinement is observed from the fact that the width of the QENS signals for ionogels are smaller than that of the pristine IL. To get further information on the local dynamics, a detailed model should be used to fit the experimental data. It has been shown that ILs possess several dynamical components, such as fast rotations of the alkyl chain, localized diffusion, and long-range diffusion $[27,28]$. However, one can also have a preliminary idea about the time scale of the dynamics by extracting the quasi-elastic component from the experimental data. The aim here is not to know the detailed quantitative information but only to get qualitative information about the dynamics to compare different samples. From this point of view, QENS spectra at $300 \mathrm{~K}$ are fitted with an elastic and a quasielastic (Lorentzian) component as described in Equation (6) (a flat background has been added to take into account the fast rotational processes). The obtained fits (with both an elastic and a quasielastic components) are in good agreement with the experimental data over the all $Q$-range and all the samples (Figure 7, Figures S2 and S3). To compare the dynamics of bulk IL, non-methylated ionogel $(x=0.25)$ and methylated ionogels with different pore sizes $(x=0.25,0.5$, and 1$)$, the HWHMs of the quasielastic width are plotted with $Q^{2}$ in Figure $8 . \Gamma(Q)$ is then fitted with the random jump diffusion model [29] to extract diffusion coefficients and elementary jump times. In that particular model, it is assumed that the particle is jumping from one site to another after spending a characteristic time $\tau_{0}$ at a previous site. The jump lengths are assumed to be random. In that case, HWHM of the quasielastic width can be represented as:

$$
H W H M=\frac{D Q^{2}}{1+D Q^{2} \tau_{0}}
$$

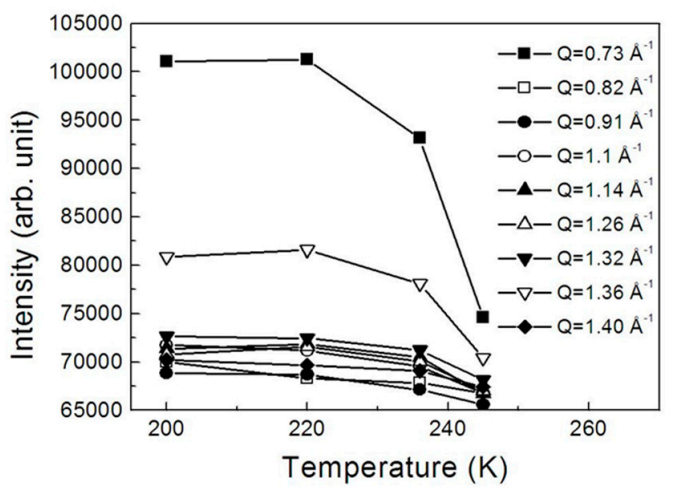

Figure 5. Intensities of different diffraction peaks with temperature for a methylated $x=0.5$ ionogel.

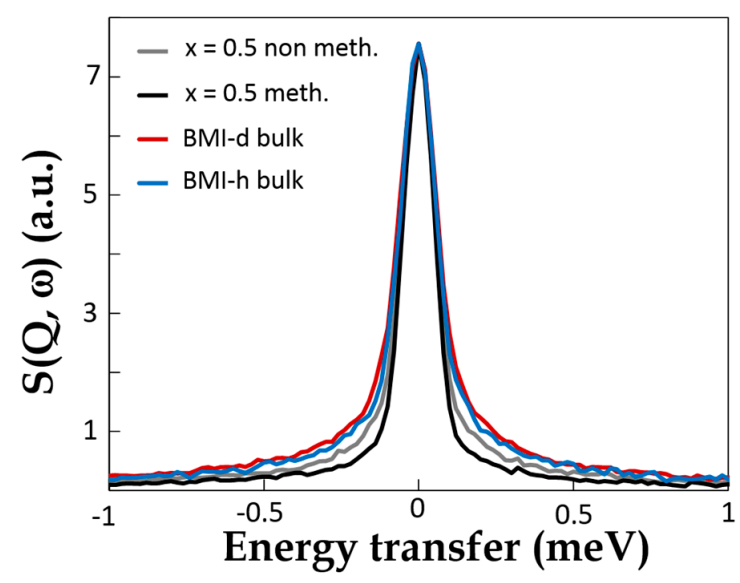

Figure 6. $S(Q, \omega)$ for various samples at $T=393 \mathrm{~K}$ recorded on the Mibemol spectrometer with an incoming wavelength of $6 \AA\left(R_{F W H M}=96 \mu \mathrm{eV}\right)$ are shown. 


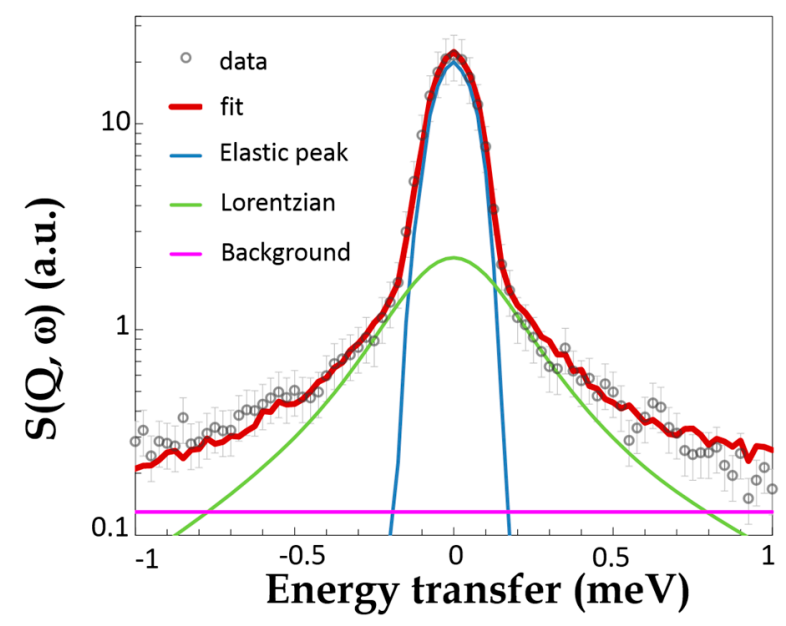

Figure 7. Quasi-elastic spectra for the methylated $x=1$ ionogel at $300 \mathrm{~K}$ at $Q=0.92 \AA^{-1}$ (incoming wavelength $\left.=5.2 \AA, R_{F W H M}=150 \mu \mathrm{eV}\right)$.

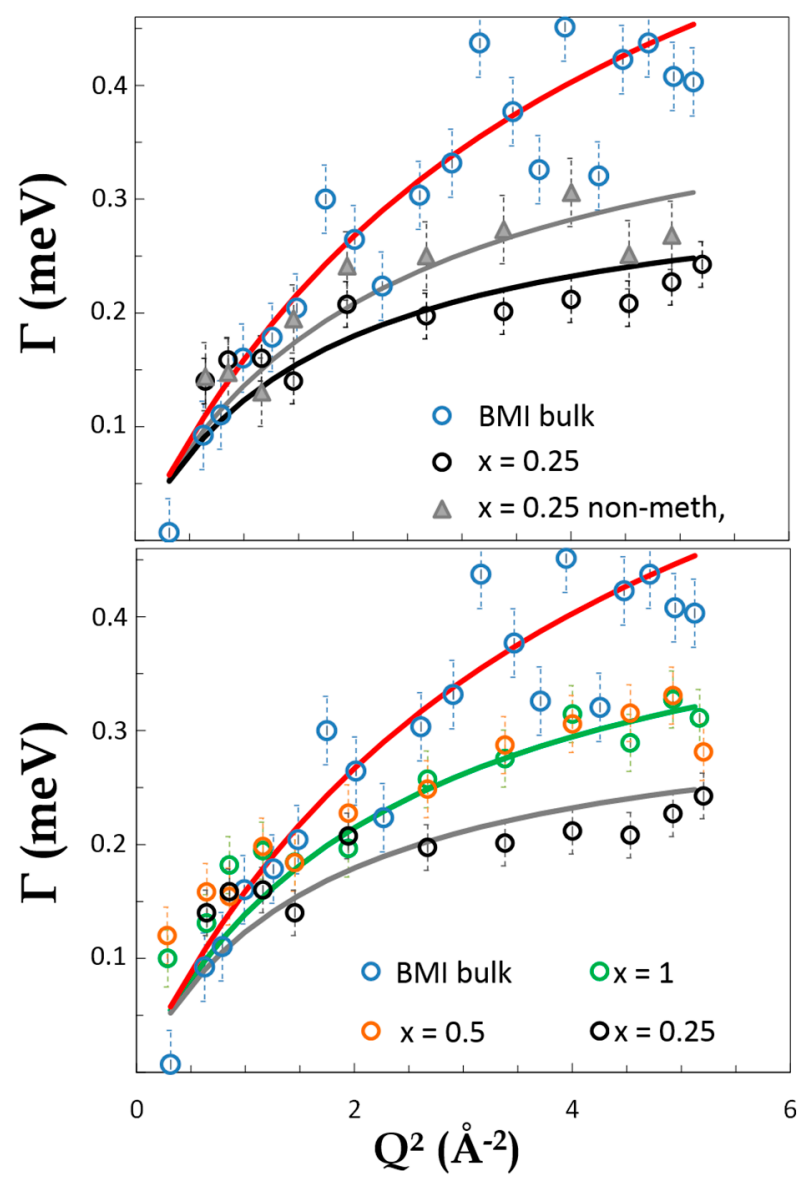

Figure 8. Variation of the widths of the quasielastic component versus $Q^{2}$ at $300 \mathrm{~K}$ for bulk BMI TFSI, $x=0.25$ methylated and non-methylated ionogel (top), and $x=0.25,0.5,1$ methylated ionogels (bottom). Solid lines are the fits with Equation (9).

By fitting the quasi-elastic widths with Equation (9), very similar diffusion coefficients are obtained for all samples with an average value of $3 \times 10^{-5} \mathrm{~cm}^{2} / \mathrm{s}$. However, characteristic times (or residence times) obtained from the fits are ranging from $0.8 \mathrm{ps}$ (for the bulk IL) to 2 ps (for the non-methylated $x=0.25$ ionogel) (Figure 8 ). These results show that the local dynamics of the methylated ionogel 
decreases moderately when the pore size decrease but remains close to the bulk IL value. The dynamics of the non-methylated $x=0.25$ ionogel is the most impacted, but the characteristic time is only increased by a factor 2.5. We note that, for this sample, the increase of the HWHM with Q is moderate and could be interpreted as a more localized motion. However, as shown below, a diffusion at the macroscopic scale is measured for all samples. We, therefore, choose to analyze all the data with the same random jump diffusion model. The decrease of diffusivity observed by QENS is attributed to the confinement of IL inside the pores, leading to the wall neighborhood effect and/or tortuosity effect. However, those effects are surprisingly low considering the volume fraction of IL confined in the ionogel, that behave mainly as bulk IL in terms of local mobility.

The self-diffusion coefficients, $D_{S}$, of cations and anions have also been measured by PFG-NMR at the micrometric scale (diffusion over few ms and $\mu \mathrm{m}$ ) (Table 2). $D_{S}$ of cations and anions measured in the ionogels are smaller than those of bulk which is consistent with the QENS analysis (at the molecular scale). The self-diffusion coefficients, $D_{S}$, decrease when the pore sizes decrease, but only by a factor 1.5 for $x=0.1$ ionogels, and a factor 4 for $x=0.25$ ionogel showing that the ionogels possess a high ionic mobility from the local to the macroscopic scale. The one order of magnitude difference between the diffusion coefficients measured by PFG-NMR and QENS has been explained in [27]. It has been shown that the ability of IL to form spontaneous nanostructures induces a scale dependence of the transport properties of the IL.

Table 2. Self-diffusion coefficients of pure IL, non-methylated (TMOS) and methylated (TMOS/MTMS) ionogels measured by PFG-NMR.

\begin{tabular}{|c|c|c|c|}
\hline Sample & $x$ & $D_{S}{ }^{1} \mathrm{H}\left(10^{-7} \mathrm{~cm}^{2} / \mathrm{s}\right)$ & $D_{S}{ }^{19} \mathrm{~F}\left(10^{-7} \mathrm{~cm}^{2} / \mathrm{s}\right)$ \\
\hline BMIM TFSI & - & 3.11 & 2.29 \\
\hline TMOS & 0.25 & 0.71 & 0.55 \\
\hline TMOS & 1 & 2.05 & 1.40 \\
\hline TMOS/MTMS & 1 & 1.70 & 1.05 \\
\hline
\end{tabular}

\section{Conclusions}

The results of the neutron scattering experiments performed on bulk IL and different ionogels gave insights at the molecular scale on the nature of phase transitions, and the structural and dynamic properties of ILs inside ionogel pores. Neutron scattering results are complementary to other studies, such as NMR spectroscopy, that probe longer time and space scales. The local mobility (mean square displacement) extracted from the quasielastic spectra indicates different phase transitions which were earlier obtained with DSC measurements and complex impedance spectroscopy, individually. Neutron diffraction carried out on one of the ionogels (Figure 4) confirmed these phase transitions and showed on this point the higher sensitivity of neutron diffraction compared to DSC. Diffusive motions of ILs in ionogels were also studied using neutron quasielastic scattering studies. The results indicate a slight confinement effect of the IL inside ionogel pores. Overall, we show that, at the molecular and micrometer scale, IL confined in the monolithic ionogel behave almost like pristine IL, thus, opening widely the use of ionogel in solid-state devices with IL properties.

Supplementary Materials: The following are available online at www.mdpi.com/1099-4300/19/4/140/s1, Figure S1: Variation of measured elastic intensity with $Q$ at different temperatures for a methylated $x=0.5$ ionogel, Figure S2: Quasielastic spectra for the bulk BMI TFSI at $300 \mathrm{~K}$ (incoming wavelength = 5.2 A), Figure S3: Quasielastic spectra for the non-methylated $x=0.25$ ionogel at $300 \mathrm{~K}$ (incoming wavelength $=5.2 \AA$ ), Table S1: Position of diffraction peak and corresponding intensity at different temperatures for ionogel with IL content of $x=0.5$.

Acknowledgments: This work was funded by the project ANR-06-NANO-003 of the Agence National de la Recherche, Paris, France.

Author Contributions: S.M. performed the QENS experiment (Mibemol); C.C. prepared the samples; C.C. and F.F. performed the DSC measurements; S.M., Q.B. and F.F. performed the QENS analysis; S.M. and Q.B. wrote the 
manuscript; R.d.O.-S. performed the neutron diffraction measurements and analysis; P.J. performed the PFG-NMR measurements; J.1.B. initiate and supervised the project; J.-M.Z. performed the QENS experiment (at IPNS) analyzed the data and supervised the project. All the authors contributed to the preparation of the manuscript.

Conflicts of Interest: The authors declare no conflict of interest.

\section{References}

1. Van Rantwijk, F.; Sheldon, R.A. Biocatalysis in ionic liquids. Chem. Rev. 2007, 107, 2757-2785. [CrossRef] [PubMed]

2. Muldoon, M.; Aki, S.; Anderson, J. Improving carbon dioxide solubility in ionic liquids. J. Phys. Chem. B 2007, 111, 9001-9009. [CrossRef] [PubMed]

3. Diaw, M.; Chagnes, A.; Carre, B.; Willmann, P.; Lemordant, D. Mixed ionic liquid as electrolyte for lithium batteries. J. Power Sources 2005, 146, 682-684. [CrossRef]

4. Ohno, H. Electrochemical Aspects of Ionic Liquids; John Wiley \& Sons: Hoboken, NJ, USA, 2005.

5. McEwen, A.; Ngo, H.; LeCompte, K. Electrochemical properties of imidazolium salt electrolytes for electrochemical capacitor applications. J. Electrochem. Soc. 1999, 146, 1687-1695. [CrossRef]

6. O'regan, B.; Grfitzeli, M. A low-cost, high-efficiency solar cell based on dye-sensitized. Nature 1991, 353, 737-740. [CrossRef]

7. Zhang, S.; Zhang, J.; Zhang, Y.; Deng, Y. Nanoconfined Ionic Liquids. Chem. Rev. 2016. [CrossRef] [PubMed]

8. Mehnert, C. Supported ionic liquid catalysis. Chem. Eur. J. 2005, 11, 50-56. [CrossRef] [PubMed]

9. Sekhon, S.; Lalia, B.; Park, J.; Kim, C. Physicochemical properties of proton conducting membranes based on ionic liquid impregnated polymer for fuel cells. J. Mater. 2006, 16, 2256-2265. [CrossRef]

10. Berrod, Q.; Ferdeghini, F.; Judeinstein, P.; Genevaz, N.; Ramos, R.; Fournier, A.; Dijon, J.; Ollivier, J.; Rols, S.; $\mathrm{Yu}, \mathrm{D}$; ; et al. Enhanced ionic liquid mobility induced by confinement in 1D CNT membranes. Nanoscale 2016, 8, 7845-7848. [CrossRef] [PubMed]

11. Néouze, M.A.; le Bideau, J.; Gaveau, P.; Bellayer, S.; Vioux, A. Ionogels, new materials arising from the confinement of ionic liquids within silica-derived networks. Chem. Mater. 2006, 18, 3931-3936. [CrossRef]

12. Guyomard-Lack, A.; Said, B.; Dupré, N.; Galarneau, A.; le Bideau, J. Enhancement of Lithium Transport by Controlling the Mesoporosity of Silica Monoliths filled by Ionic Liquids. New J. Chem. 2016, 40, 4269-4276. [CrossRef]

13. Le Bideau, J.; Gaveau, P.; Bellayer, S.; Néouze, M.; Vioux, A. Effect of confinement on ionic liquids dynamics in monolithic silica ionogels: ${ }^{1} \mathrm{H}$ NMR study. Phys. Chem. Chem. Phys. 2007, 9, 5419-5422. [CrossRef] [PubMed]

14. Bonhôte, P.; Dias, A.-P.; Papageorgiou, N.; Kalyanasundaram, K.; Grätzel, M. Hydrophobic, Highly Conductive Ambient-Temperature Molten Salts. Inorg. Chem. 1996, 35, 1168-1178. [CrossRef] [PubMed]

15. Néouze, M.; Le Bideau, J.; Vioux, A. Versatile heat resistant solid electrolytes with performances of liquid electrolytes. Prog. Solid State Chem. 2005, 33, 217-222. [CrossRef]

16. Inelastic Time-of-Flight Spectrometer MIBEMOL. Available online: http://www-llb.cea.fr/spectros/pdf/ mibemol-1lb.pdf (accessed on 21 March 2017).

17. Kolesnikov, A.; Zanotti, J.; Loong, C. Scientific Review: Spectroscopy at IPNS: Recent Instrumental Upgrade and Scientific Highlights. Neutron News 2004, 15, 19-22. [CrossRef]

18. Bée, M. Quasielastic Neutron Scattering: Principles and Applications in Solid State Chemistry, Biology, and Materials Science; Adam Hilger: Bristol, PA, USA, 1988.

19. G6-1. Available online: www-llb.cea.fr/fr-en/pdf/g61-llb.pdf (accessed on 21 March 2017).

20. Kanakubo, M.; Hiejima, Y.; Minami, K.; Aizawa, T. Melting point depression of ionic liquids confined in nanospaces. Chem. Commun. 2006, 17, 1828-1830. [CrossRef] [PubMed]

21. Bicout, D.; Zaccai, G. Protein flexibility from the dynamical transition: A force constant analysis. Biophys. J. 2001, 80, 1115-1123. [CrossRef]

22. Canongia Lopes, J.N.A.; Pádua, A.A.H. Nanostructural organization in ionic liquids. J. Phys. Chem. B 2006, 110, 3330-3335. [CrossRef] [PubMed]

23. Triolo, A.; Russina, O.; Bleif, H.-J.; Di Cola, E. Nanoscale segregation in room temperature ionic liquids. J. Phys. Chem. B 2007, 111, 4641-4644. [CrossRef] [PubMed] 
24. Angell, C.; Byrne, N.; Belieres, J. Parallel developments in aprotic and protic ionic liquids: Physical chemistry and applications. Acc. Chem. Res. 2007, 40, 1228-1236. [CrossRef] [PubMed]

25. Guyomard-Lack, A.; Delannoy, P.-E.; Dupré, N.; Cerclier, C.V.; Humbert, B.; le Bideau, J. Destructuring ionic liquids in ionogels: Enhanced ionic liquid properties for solid devices. Phys. Chem. Chem. Phys. 2014, 16, 23639-23645. [CrossRef] [PubMed]

26. Xu, W.; Angell, C. Solvent-free electrolytes with aqueous solution-like conductivities. Science 2003, 302, 422-425. [CrossRef] [PubMed]

27. Ferdeghini, F.; Berrod, Q.; Zanotti, J.-M.; Judeinstein, P.; Sakai, V.G.; Czakkel, O.; Fouquet, P.; Constantin, D.; Rols, S.; Yu, D.; et al. Nanostructuration of ionic liquids: Impact on the cation mobility. A multi-scale study. Nanoscale 2017, 9, 1901-1908. [CrossRef] [PubMed]

28. Burankova, T.; Reichert, E.; Fossog, V.; Hempelmann, R.; Embs, J.P. The dynamics of cations in pyridinium-based ionic liquids by means of quasielastic- and inelastic neutron scattering. J. Mol. Liq. 2014, 192, 199-207. [CrossRef]

29. Hall, P.L.; Ross, D.K. Incoherent neutron scattering functions for random jump diffusion in bounded and infinite media. Mol. Phys. 1981, 42, 673-682. [CrossRef]

(C) 2017 by the authors. Licensee MDPI, Basel, Switzerland. This article is an open access article distributed under the terms and conditions of the Creative Commons Attribution (CC BY) license (http:/ / creativecommons.org/licenses/by/4.0/). 